\title{
Full Tomographic Reconstruction of 2D Vector Fields using Discrete Integral Data
}

\author{
Maria Petrou ${ }^{1, *}$ AND Archontis GiannaKidis ${ }^{2}$ \\ ${ }^{1}$ Department of Electrical and Electronic Engineering, Imperial College, London, SW7 2AZ, UK \\ ${ }^{2}$ Faculty of Engineering and Physical Sciences, University of Surrey, Guildford, GU2 7XH, UK \\ *Corresponding author: maria.petrou@imperial.ac.uk
}

\begin{abstract}
Vector field tomography is a field that has received considerable attention in recent decades. It deals with the problem of the determination of a vector field from non-invasive integral data. These data are modelled by the vectorial Radon transform. Previous attempts at solving this reconstruction problem showed that tomographic data alone are insufficient for determining a $2 \mathrm{D}$ band-limited vector field completely and uniquely. This paper describes a method that allows one to recover both components of a 2D vector field based only on integral data, by solving a system of linear equations. We carry out the analysis in the digital domain and we take advantage of the redundancy in the projection data, since these may be viewed as weighted sums of the local vector field's Cartesian components. The potential of the introduced method is demonstrated by presenting examples of vector field reconstruction.
\end{abstract}

Keywords: vector field tomography; radon transform; inverse problems

Received 17 September 2009; revised 3 June 2010

Handling editor: Suchi Bhandarkar

\section{INTRODUCTION}

In recent years Hough transform [1] and the related Radon transform [2] have received much attention. Theoretical ideas found in Radon's early work apply in conventional tomographic reconstruction, very common in medical imaging [3]. Functions that are reconstructed by using traditional tomography are scalar functions describing, e.g. absorption or scattering properties. However, over the last few decades there has been a growing demand for similar techniques that would perform tomographic reconstruction of a vector field, rather than a scalar one, when having integral information. The problem of recovering a vector field from its line integrals has generally been regarded as an under-determined problem. This seems to be clear from the fact that a scalar function is determined uniquely from its Radon transform, whereas a vector field requires two (in 2D) or three (in 3D) component functions to be determined. Several applications of vector field tomography have been considered in the literature. These include: blood flow imaging $[4,5]$; fluid mesoscale velocity imaging in ocean acoustic tomography [6-8]; fluid-flow imaging [9-16]; electric field imaging in Kerr materials [17-19]; imaging of the component of the gradient of the refractive index field, which is transversal to the beam, in Schlieren tomography [14]; velocity field imaging of heavy particles in plasma physics [20]; density imaging in supersonic expansions and flames in beam deflection optical tomography [21]; non-destructive stress distribution imaging of transparent specimens in photoelasticity [22,23]; determination of temperature distributions and velocity vector fields in furnaces [24]; and magnetic field imaging in Tokamak in polarimetric tomography [25].

Using the physics of the applications mentioned above, it can be shown that, in each case, the acquired measurements reduce to an integral transform of the investigated vector field along integration lines, the vectorial Radon transform. When we try to investigate planar vector fields in bounded domains, two classes of the vectorial Radon transform that model the tomographic measurements, arise, depending on the interaction between the obtained measurements and the investigated vector field. The first type of the line integral transform $J_{1}$ is

$$
J_{1}=\int_{L} \overline{\mathbf{f}}(x, y) \cdot \hat{\mathbf{s}} \mathrm{d} s=\int_{L} f_{\|} \mathrm{d} s,
$$

where $\overline{\mathbf{f}}(x, y)$ is the planar vector field under investigation, $\hat{\mathbf{s}}$ is the unit vector along the integration (measurement) line $L$, 
$\mathrm{d} s$ is an element of path length along this line, $\cdot$ is the symbol for dot product and $f_{\|}$is the component of $\overline{\mathbf{f}}(x, y)$ along $L$. From equation (1) we may deduce that only the component of $\overline{\mathbf{f}}(x, y)$ along line $L$ is observed in this type of measurement (longitudinal measurements). The second class of line integral transforms $J_{2}$ is used to model tomographic measurements that collect information from the component of the investigated vector field perpendicular to the measurement line (transversal measurements):

$$
J_{2}=\int_{L} \overline{\mathbf{f}}(x, y) \cdot \hat{\boldsymbol{\rho}} \mathrm{d} s=\int_{L} f_{\perp} \mathrm{d} s .
$$

Here $\hat{\rho}$ is the unit vector perpendicular to the line of integration $L$ and $f_{\perp}$ is the component of $\overline{\mathbf{f}}(x, y)$ transverse to $L$. The integral transforms of equations (1) and (2) (which are the two types of vectorial Radon transform for planar vector fields) are a natural generalization of the classical Radon transform to vector fields.

During the short history of 2D vector field tomography, many investigators attempted to solve the reconstruction problem $[13,14,22,26]$, but the result has always been the same: only one component of the vector field could be recovered from the tomographic measurements. The component that could be recovered was either the curl-free (irrotational) part or the divergence-free (solenoidal) part, depending on the physical principle of the measurements, namely the relation between the obtained set of measurements and the investigated vector field. One possible solution to this problem would be to collect data using both types of relation between the measurements and the examined vector field for every application. Indeed, such an amount of information would be sufficient to allow the full reconstruction of the vector field as Braun and Hauck demonstrated in [14]. Unfortunately, there are only very few specialized applications (mainly in optics), where it is physically realizable to have all these measurements available. Moreover, as Norton showed in [13], we may have a full reconstruction based only on longitudinal measurements, as long as, apart from the longitudinal measurements, supplementary information about the vector field, especially boundary conditions and a priori information about the source distribution, is available as well. In addition, Rouseff and Winters showed in [27] that a 2D vector field reconstruction based on boundary data is possible for scattering geometries. However, the model they used for the available scattering measurements is different from the integral transforms of equations (1) and (2).

All the conclusions concerning 2D vector field tomography described above were drawn from work that was carried out in the continuous domain. In this paper we consider the problem in the digital domain and consider only band-limited fields. We wish to reconstruct the field in specific sampling points arranged in a grid, finite in number. Then, there is a lot of redundancy in the data. Although the available measurements are projections of one component of the field (along or perpendicular to the direction of the tracing line), we may use many line orientations passing through every point and then view their recordings as weighted sums of the local vector field's Cartesian components. We may then exploit the redundancy in the data to recover the vector field at all sampling points of the $2 \mathrm{D}$ domain. The extension to 3D is straightforward, limited only by the number of simultaneous linear equations one can solve.

This paper is organized as follows. In Section 2 we present our methodology. In Section 3 we define the way we sample the parametric space. In Section 4 we present an example application, where a static electric field is reconstructed from voltage measurements only on the boundary of the field. This example is chosen because from Coulomb's law we can compute exactly the ground truth and thus evaluate the proposed methodology. In Section 5 we examine the effect of noise on the reconstruction of the vector field. We conclude in Section 6.

\section{THE PROPOSED METHODOLOGY}

The whole treatment in this section is performed in the digital domain. The bounded 2D domain, within which we want to recover the vector field, is divided into tiles of finite size. The values of the components of the vector field in every such tile are the values of the components of the field at the centre of this tile, namely the sampling point. Hence, our formulation of the vector field reconstruction problem is in terms of Cartesian vector components required at these sampling points of the $2 \mathrm{D}$ domain.

In order to achieve the full vector field recovery, we exploit the redundancy in the integral data, since these data may be viewed as weighted versions of the local vector field's Cartesian components. The line-integral data are obtained from a finite number of points, that reside on the boundary of the 2D domain, where measurements are made. These points are assumed to be the locations of ideal point sensors. The solution to the reconstruction problem is based only on the projection data along lines defined by the finite number of measurement points.

The ultimate objective of this analysis is to recover both components of the vector field at every sampling point of the bounded 2D domain by solving a system of linear equations. In this study, in order to form this system of linear equations, we assume that a pair of sensors measures only the integral of the component of the vector field along the line defined by these sensors. Hence, the integral transform that models the process of data acquisition is given by equation (1). By using all available line-integrals, we obtain the required system of linear equations, the solution of which is expected to give all the components of the vector field at all sampling points of the 2D domain. Since we have two unknowns per sampling point of the $2 \mathrm{D}$ space, namely the components $\left(f_{x}, f_{y}\right)$ of the vector field, the number of the available equations has to be selected so that the number of equations exceeds the number of the unknowns. By solving then the system of equations in the least-squares error sense, we expect to achieve robustness in the 
solution. Such a constraint may be easily satisfied. As a gross estimate, we may assume that if we sample the space by $N \times N$ grid points, where we wish to estimate the field, and assume the $4 N$ sampling points on the perimeter to represent the sensors, we can form $(4 N) ! /[(4 N-2) ! 2 !]$ equations by considering all possible combinations of the sensors in pairs. We have $2 N^{2}$ unknowns and $2 N(4 N-1)=8 N^{2}-2 N$ equations, a far larger number of equations than unknowns.

Next, we must translate into the digital domain the integration expressed by equation (1) in the continuous domain. The integrals of the vector field along the tracing lines have to be expressed in terms of the components of the field at the sampling points of the 2D grid. To do that we follow the methodology used in [28-30] for the calculation of the trace transform.

Let us assume that we have the digitized square $2 \mathrm{D}$ domain shown in Fig. 1 and we wish to recover vector field $\overline{\mathbf{f}}(x, y)$ at the centre of every tile of this space. The origin of the axes of the coordinate system is chosen to be at the centre of the domain. The length of each side of the square domain is taken to be equal to $2 U$. The size of the tiles with which we sample the $2 \mathrm{D}$ domain is $P \times P$, so that $2 U / P$ is an integer. Then the total number of tiles of the digitized domain is $4 U^{2} / P^{2}(=2 U / P \times 2 U / P)$ and the overall number of the unknowns is $X \equiv 8 U^{2} / P^{2}$, since we have two unknown components per tile of the $2 \mathrm{D}$ space. Moreover, we assume that there are ideal point sensors that reside on predetermined and regularly placed positions along the border of this domain. These positions are the middle points of the boundary edges of all boundary tiles. Hence, $8 U / P$ $(=4 \times 2 U / P)$ sensors, in total, are located along the boundary of the domain of Fig. 1.

Let us consider a line segment $A B$ between two such sensors, chosen arbitrarily, crossing this domain as shown in Fig. 1. Next, we show how the available line-integral measurement $J_{i}$

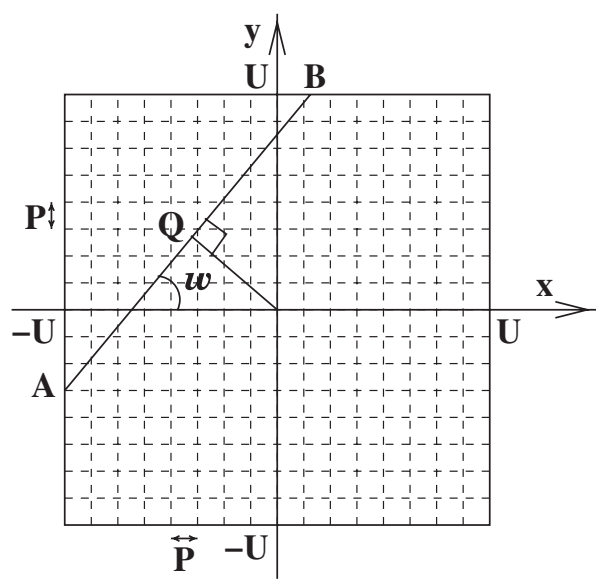

FIGURE 1. A line segment between two boundary sensors that reside at points $A$ and $B$. The angle between the line segment and the positive direction of the $x$-axis is $w$. The size of the tiles, with which we sample the 2D space, is $P \times P$. Point $Q$ is the foot of the normal from the origin of the axes to the line segment. between $A$ and $B$, which is described by equation (1), gives rise to a linear equation. The known coordinates of points $A$ and $B$ are $\left(x_{A}, y_{A}\right)$ and $\left(x_{B}, y_{B}\right)$, respectively. Therefore, the equation of line $A B$ is

$$
y=\lambda x+\beta,
$$

where

$$
\lambda \equiv \frac{y_{B}-y_{A}}{x_{B}-x_{A}} \quad \text { and } \quad \beta \equiv y_{A}-\frac{y_{B}-y_{A}}{x_{B}-x_{A}} x_{A} .
$$

The unit vector $\hat{\mathbf{s}}$ along the line (and with direction from $A$ to $B)$ is

$$
\hat{\mathbf{s}}=\cos w \hat{\mathbf{x}}+\sin w \hat{\mathbf{y}},
$$

where $w=\arctan \lambda$ is the angle between the line and the positive direction of the $x$-axis (Fig. 1). The next step is to perform a sampling of the line segment. The starting point of this sampling will be the foot of the normal of this line from the origin of the axes (point $Q$ in Fig. 1). The coordinates of the starting point $Q$ for the calculations along the line are

$$
x_{Q}=-\frac{\beta}{(\lambda+1 / \lambda)}, \quad y_{Q}=-\frac{1}{\lambda} x_{Q} .
$$

The sampling along the line section will be performed on either side of $Q$ and we assume that the sampling step is $\Delta s$. The maximum number of sampling intervals that we can fit in this line section is determined by the intersection points between the line and the border of the 2D domain. The distances between the starting point $Q$ and intersection points $A$ and $B$ are $d_{A}$ and $d_{B}$, respectively. Consequently, the numbers $l_{A}$ and $l_{B}$ of $\Delta s$, that we may fit in the line segment between the foot of the normal $Q$ and the boundary points $A$ and $B$, respectively, are

$$
l_{A}=\left\lfloor\frac{d_{A}}{\Delta s}\right\rfloor, \quad l_{B}=\left\lfloor\frac{d_{B}}{\Delta s}\right\rfloor,
$$

where $L\rfloor$ is the symbol for the floor operator. Therefore, the sampling points we shall consider along the line segment will have the coordinates

$$
x_{l}=x_{Q}+l x_{\mathrm{inc}}, \quad y_{l}=y_{Q}+l y_{\mathrm{inc}} \quad \text { for } l \in\left[-l_{A}, l_{B}\right],
$$

where the increments $x_{\text {inc }}$ and $y_{\text {inc }}$ of the coordinates, between successive sampling points, are given by

$$
x_{\mathrm{inc}}=\Delta s \cos w, \quad y_{\mathrm{inc}}=\Delta s \sin w .
$$

The total number of sampling points along the line segment is $l_{A}+l_{B}+1$.

After having worked out the coordinates of the sampling points of the line, we must assign them values from the vector field. We use nearest neighbour interpolation for that. Hence, we need to determine for each sampling point of the line, the tile the centre point of which is its nearest neighbour. For this purpose, we use the integer coordinates $(i, j)$ with $i, j=1, \ldots, 2 U / P$, of each tile of the 2D domain, as shown in Fig. 2. 


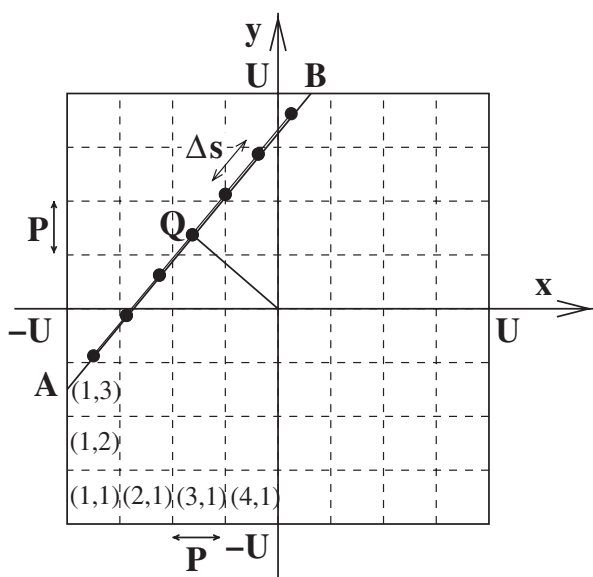

FIGURE 2. The integer coordinates $(i, j)$ with $i, j=1, \ldots, 2 U / P$ of the tiles of the $2 \mathrm{D}$ domain. Also shown is an example of sampling a line segment with sampling step $\Delta s$.

Then tile $(i, j)$, which corresponds to a sampling point $\left(x_{l}, y_{l}\right)$, is identified by using the formulae

$$
i=\left\lceil\frac{x_{l}+U}{P}\right\rceil, \quad j=\left\lceil\frac{y_{l}+U}{P}\right\rceil,
$$

where \lceil\rceil is the ceiling operator.

The next step is to form the equation that corresponds to the line-integral measurement $J_{i}$ between sensors at points $A$ and $B$. This may be achieved by considering the sampling points of segment $A B$ as the centres of linear segments of length $\Delta s$, apart from the sampling points with $l=-l_{A}$ and $l=l_{B}$, which are special cases. Along each segment of length $\Delta s$, the vector field is assumed constant, equal to the assigned value of the vector field at the corresponding sampling point of the line. We may, then, convert the integral of equation (1) into a sum by projecting the value of the field at each sampling point of the line onto the vector that represents the direction of the line:

$$
J_{i}=\sum_{l=-l_{A}+1}^{l_{B}-1} \overline{\mathbf{f}_{l}} \cdot \overline{\Delta \mathbf{s}}+\overline{\mathbf{f}}_{l A} \cdot \hat{\mathbf{s}} \Delta_{A}+\overline{\mathbf{f}}_{l B} \cdot \hat{\mathbf{s}} \Delta_{B} .
$$

Here $\overline{\mathbf{f}}_{l}=\left(f_{x l}, f_{y_{l}}\right), \overline{\mathbf{f}}_{l A}=\left(f_{x l A}, f_{y_{l A}}\right)$ and $\overline{\mathbf{f}}_{l B}=\left(f_{x l B}, f_{y_{l B}}\right)$ are the (unknown) assigned vector field values at the sampling points $l, l_{A}$ and $l_{B}$, respectively, $\overline{\Delta \mathbf{s}}=\Delta s \hat{\mathbf{s}}=\Delta s(\cos w \hat{\mathbf{x}}+$ $\sin w \hat{\mathbf{y}})$, and

$$
\Delta_{A}=\frac{\Delta s}{2}+d_{l A}, \quad \Delta_{B}=\frac{\Delta s}{2}+d_{l B},
$$

where $d_{l A}$ is the distance between the sampling point with $l=-l_{A}$ and the boundary point $A$, and $d_{l B}$ is the distance between the sampling point with $l=l_{B}$ and the boundary point $B$. In order to obtain the system of linear equations, the solution of which will give the components of the unknown vector field $\overline{\mathbf{f}}(x, y)$ at all sampling points of the $2 \mathrm{D}$ domain, we repeat the procedure described above for all possible pairs of boundary point sensors. Under the assumption that there are $2 U / P$ ideal point sensors on each side of the boundary of the domain of Fig. 1, and since line-integral data obtained from pairs of sensors on the same side of the boundary of the square are not useful, the number of equations is

$$
G \equiv \frac{24 U^{2}}{P^{2}}\left[=\frac{2 U}{P}\left(3 \times \frac{2 U}{P}+2 \times \frac{2 U}{P}+1 \times \frac{2 U}{P}\right)\right] .
$$

Here we have taken care that each line segment $A B$ is counted only once.

To summarize, our formulation of the vector field reconstruction problem may be written as

$$
\mathbf{g}=\mathbf{C x},
$$

where $\mathbf{g} \in \mathbb{R}^{G \times 1}$ is the vector that contains the available projection measurements wrapped into a vector, $\mathbf{x} \in \mathbb{R}^{X \times 1}$ is the set of the components of the vector field to be reconstructed at all sampling points of the $2 \mathrm{D}$ digitized domain written as a vector and $\mathbf{C} \in \mathbb{R}^{G \times X}$ is the system matrix containing the weight factors between each of the components of the vector field at every reconstruction point and each of the corresponding tracing line orientations from the set of measurements. System matrix C is obtained from the analysis described above. Moreover, it is $G>X$, in accordance with our intention to take advantage of the redundancy in the line-integral data, and, therefore, we have to deal with an over-determined system of linear equations.

\section{UNIFORM RADON DOMAIN SAMPLING}

The reconstruction method described in the previous section is based on linear algebra. This approach expresses the tomographic vector field reconstruction problem in terms of a system of linear equations. However, there is a duality between the Radon transform scheme and this matrix formalism. Hence, solving the above described system of linear equations is equivalent to inverting the vectorial Radon transform. According to the theory of the Radon transform [3], a necessary requirement for producing accurate reconstructions is to sample uniformly the Radon domain parameter space, defined by the length of the normal to the tracing line $\rho$ and the angle $\theta$ this normal forms with the positive $x$-axis (see Fig. 3).

Placing our sensors, however, uniformly along the border of the reconstruction grid does not produce a uniform sampling of the parameter space. Instead, we must follow [28-30] and uniformly sample parameters $\rho$ and $\theta$. From the values of these parameters, then the locations of the sensors along the boundary of the domain should be determined. Therefore, we sample the parameters $\rho$ and $\theta$ with the steps $\Delta \rho$ and $\Delta \theta$, respectively:

$$
\begin{aligned}
\rho_{r}=r \Delta \rho, & r=0,1, \ldots, R-1, \\
\theta_{t}=t \Delta \theta, & t=0,1, \ldots, T-1,
\end{aligned}
$$




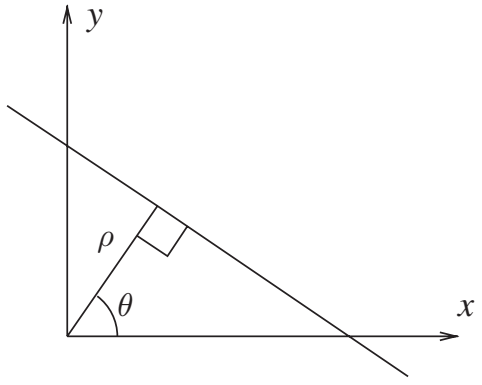

FIGURE 3. The two parameters $\rho$ and $\theta$ used to define a tracing line. These variables are the coordinates of the Radon domain.

where $R$ and $T$ are the total number of samples used for the parameters $\rho$ and $\theta$, respectively. It is a common practice in such cases to consider angle $\theta$ in the range $[0, \pi]$ while $\rho$ is allowed to take positive and negative values, with the negative values corresponding to lines with $\theta>\pi$. This convention is used in order to take into consideration the direction of the scanning line. In our problem, however, each line is considered only once, as direction does not matter. Thus, we allow the parameter $\theta$ to range between 0 and $2 \pi$, while $\rho$ ranges between 0 and $\rho_{\max }$, where $\rho_{\max }$ is half the maximum diagonal of the $2 \mathrm{D}$ domain we consider. We consider all possible pairs of values of these two parameters. Each pair $\left(\rho_{l}, \theta_{l}\right)$ with $l=1, \ldots, R T$ describes a tracing line with the following equation:

$$
\rho_{l}=x \cos \theta_{l}+y \sin \theta_{l}, \quad l=1, \ldots, R T .
$$

This tracing line corresponds to a line-integral measurement or, equivalently, to a linear equation of the required system of equations. In order to obtain the boundary positions, where the two sensors that will collect this measurement need to be placed, we must find the points where this line intersects the domain boundaries. If we assume that we have the same rectangle of interest of Fig. 1, as in Section 2, the borders are segments of the lines $x= \pm U$ and $y= \pm U$. Therefore, for tracing lines, that are not parallel to either the $x$ or the $y$-axis, the four intersection points $\left(U, y_{1}\right),\left(-U, y_{2}\right),\left(x_{1}, U\right)$ and $\left(x_{2},-U\right)$, between the (a) Recovered field
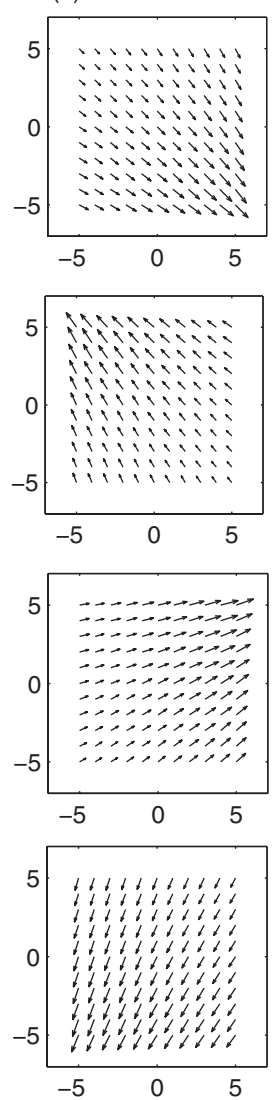

(b) Theoretical field
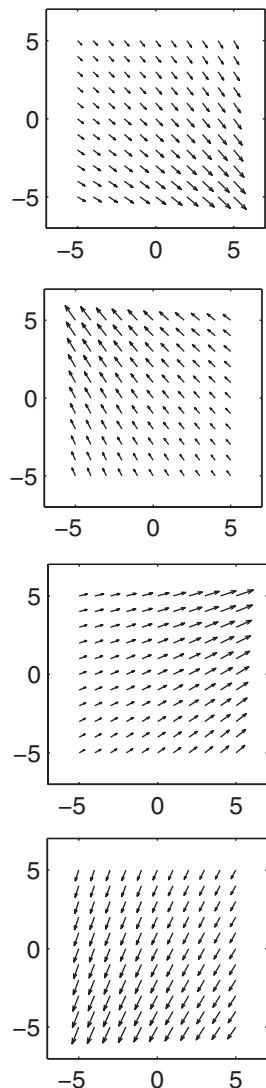

(c) Magnitude error (\%)
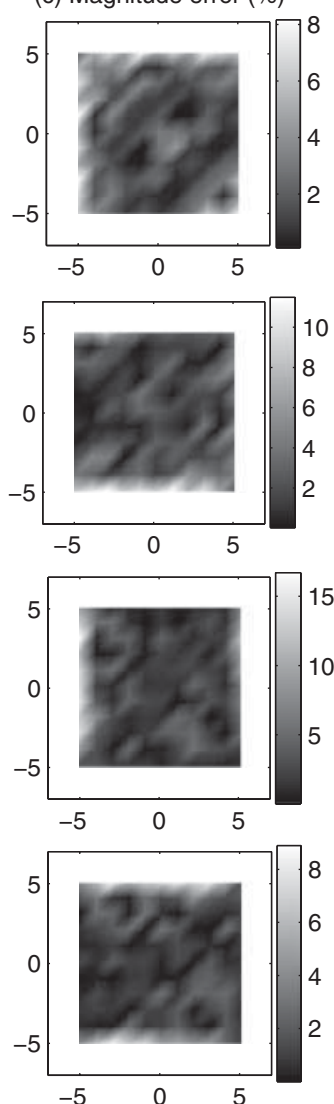

(d) Angular error $\left({ }^{\circ}\right)$
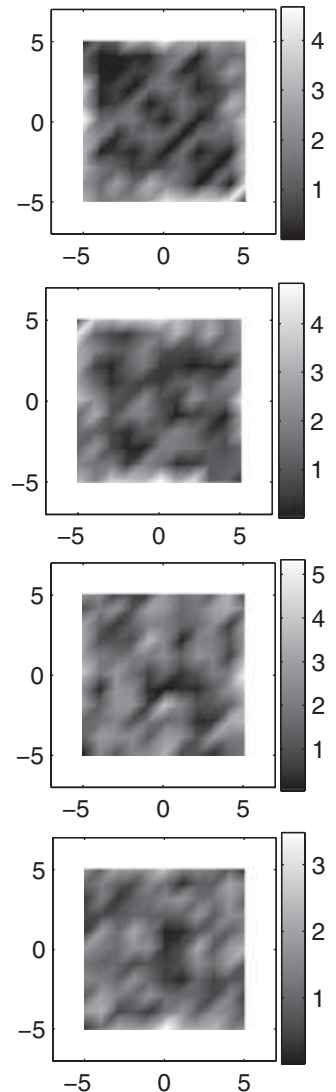

FIGURE 4. Simulation results for the cases when the reconstruction was based on integral data, the corresponding tracing lines of which have uniform distribution in the Radon domain with $\Delta \rho=1$ and $\Delta \theta=2^{\circ}$. The location of the source of the electric field was (from top to bottom) at $(19,-19),(-16,21),(24,11.5)$ and $(-19,-40)$ : (a) the recovered vector field; (b) the electric field as computed from Coulomb's law; (c) the relative magnitude difference between the above two fields (\%); (d) the absolute angular difference (in degrees) between the directions of the recovered and the true field. 
considered measurement line and the lines $x= \pm U, y= \pm U$, have to be obtained as follows:

$$
\begin{aligned}
& y_{1}=\frac{\rho_{l}}{\sin \theta_{l}}-U \cot \theta_{l}, \\
& y_{2}=\frac{\rho_{l}}{\sin \theta_{l}}+U \cot \theta_{l}, \\
& x_{1}=\frac{\rho_{l}}{\cos \theta_{l}}-U \tan \theta_{l}, \\
& x_{2}=\frac{\rho_{l}}{\cos \theta_{l}}+U \tan \theta_{l} .
\end{aligned}
$$

Out of the estimated four numbers $y_{1}, y_{2}, x_{1}$ and $x_{2}$, we select the two of them with the absolute value less than $U$. There will always exist two such numbers for tracing lines that lie within the rectangle of interest. These two numbers identify the two of the four pairs $\left(U, y_{1}\right),\left(-U, y_{2}\right),\left(x_{1}, U\right)$ and $\left(x_{2},-U\right)$ that give the coordinates of the two intersection points between the tracing line $\left(\rho_{l}, \theta_{l}\right)$ and the border of the domain of interest, where the two ideal sensors should reside. For tracing lines that are parallel to the $x$-axis, i.e. lines with $\theta_{l}=\pi / 2$ or $3 \pi / 2$, the equation of the line is $y= \pm \rho_{l}$ and the two intersection points are $\left(U, \pm \rho_{l}\right)$ and $\left(-U, \pm \rho_{l}\right)$, respectively. Likewise, for tracing lines that are parallel to the $y$-axis, i.e. lines with $\theta_{l}=0$ or $\pi$, the equation of the line is $x= \pm \rho_{l}$ and the two intersection points are $\left( \pm \rho_{l}, U\right)$ and $\left( \pm \rho_{l},-U\right)$, respectively.

Having found the coordinates where the ideal sensors should be placed, the system of linear equations is formed in a way similar to the one presented in Section 2.

As a consequence of the necessity of uniform sampling in the parameter space, the sensors can no longer be regularly placed along the border of the domain. Also, the number of the required sensors is now significantly higher, theoretically twice the number of the line-integrals that are considered.

\section{AN EXAMPLE: ELECTRIC FIELD IMAGING}

In this section we consider the case where the vector field that we want to recover is the electric field created by a static charge. There are many ways to recover the electric field from boundary data. However, here we use the electric field only to demonstrate our method. In order to satisfy the condition of a band-limited field and avoid problems with singularities, we place the source of the vector field that we aim to recover outside the bounded 2D (a) Magn. err. (\%)
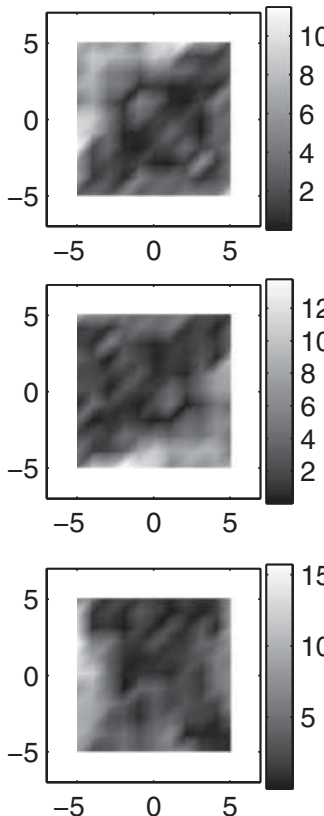

(b) Ang. err. $\left({ }^{\circ}\right)$

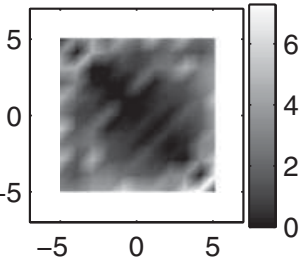

(c) Magn. err. (\%)

(d) Ang. err. $\left({ }^{\circ}\right)$
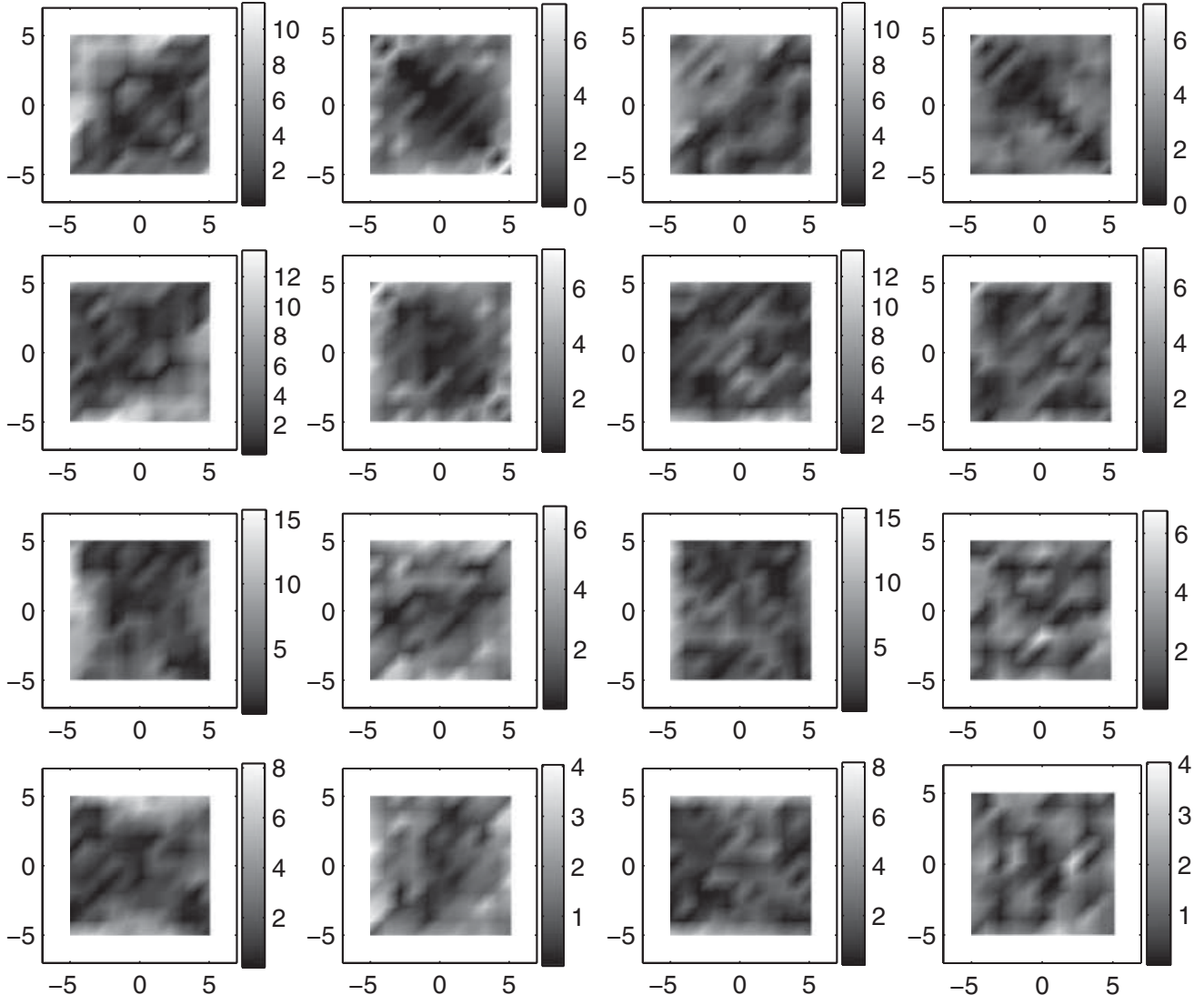

FIGURE 5. Left two columns: error in the reconstructions when the sensors are placed uniformly along the boundary. Right two columns: errors in the reconstruction when the sampling is done uniformly in the Radon domain with $\Delta \rho=1$ and $\Delta \theta=3^{\circ}$. The location of the source of the electric field was (from top to bottom) at $(19,-19),(-16,21),(24,11.5)$ and $(-19,-40)$. 
area. In a real physical system, we do not expect to have to deal with real singularities anyway. Again we stress that the problem we solve is intentionally kept simple in order to demonstrate the method. Hence, instead of avoiding singularities by using a realistic version of Coulomb's law for sources of finite size, we place the source outside the domain of interest and make it infinitesimally small.

We assume that the sensors we use measure the local potential of the field. For a static electric field, every voltage difference between any two points is the line-integral of the electric field projected along the line that connects the two points. We may then say that these voltage differences give the vectorial Radon transform of the electric field.
For our experiments, we employed the digital square domain of Fig. 1 and chose $2 U=11$ as the domain size and $P=1$ as the tile size. Hence, the domain consists of 121 tiles and the number of the unknowns (the $E_{x}$ and $E_{y}$ components of the field for every tile of the domain) is 242 . Four different cases for the location of a single source vector field are first reported. We relied entirely on a number of voltage differences obtained between ideal point sensors (electrodes) that lie on the boundary of this domain to carry out the electric field reconstruction. In order to acquire the simulated potential measured by every sensor, we used Coulomb's law. The sampling step along the tracing lines was set to 1 . We used uniform sampling in the parameter space, using the sampling steps recommended in
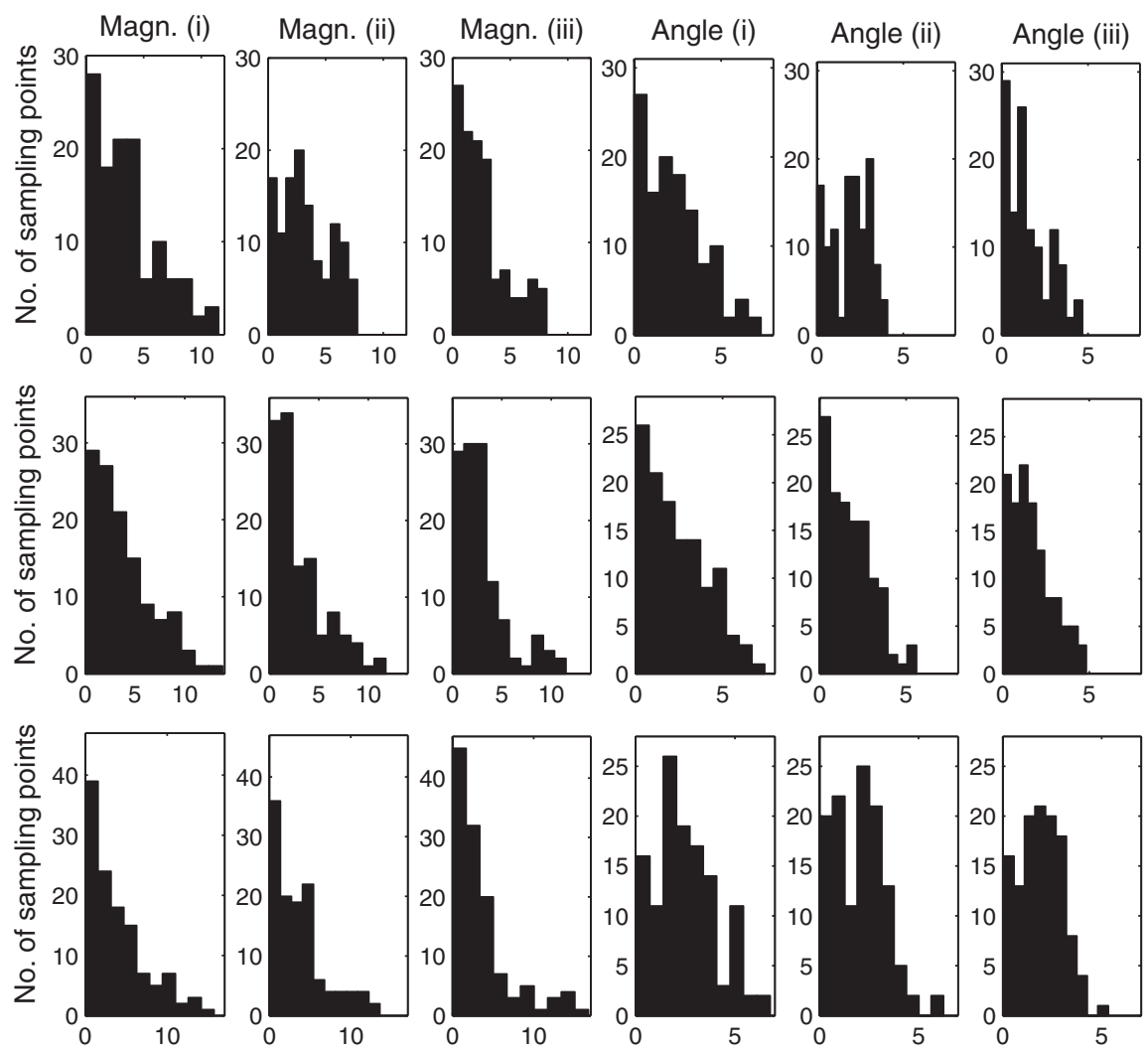

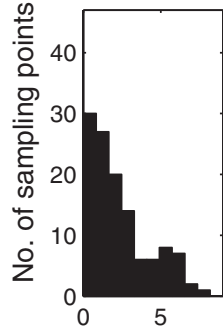

$(\%)$

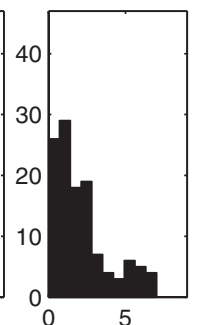

(\%)

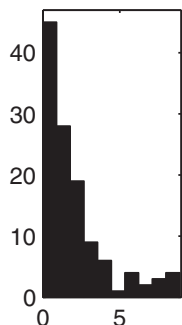

$(\%)$

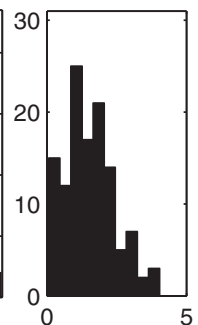

$\left({ }^{\circ}\right)$

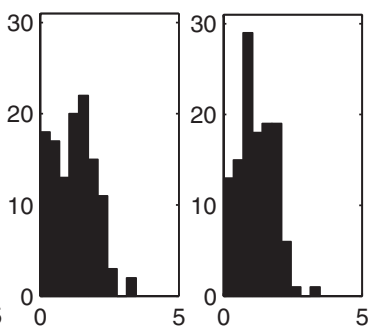

$\left({ }^{\circ}\right)$

$\left({ }^{\circ}\right)$

FIGURE 6. Left three columns: the relative errors in magnitude for the cases (i) uniform sampling in $(x, y)$; (ii) uniform sampling in ( $\rho, \theta)$ with $\Delta \rho=1$ and $\Delta \theta=3^{\circ}$; (iii) uniform sampling in $(\rho, \theta)$ with $\Delta \rho=1$ and $\Delta \theta=2^{\circ}$. Right three columns: the error in vector field orientation for the same cases. The location of the source of the electric field was (from top to bottom) at $(19,-19),(-16,21),(24,11.5)$ and $(-19,-40)$. We note that the histograms of the first and the fourth columns have longer tails towards higher values. 
$[29,30]$, namely $\Delta \rho=1$ and $\Delta \theta=2^{\circ}$. This yielded an over-determined system of $6 \times 180=1080$ equations. The solution of this system of equations was obtained by applying the least-squares method. The Householder orthogonalization method [31], which is a numerically useful procedure in order to solve mean square value problems for cases where the condition number of the matrix of coefficients is large [32], was also tested for our reconstruction problem. However, the results we obtained were identical with the results we obtained using the least-squares method. Moreover, it must be noted that since the residual we computed by using the least-squares method was not large when compared with the solution vector, there was no need to use the Cholesky method [33]. The reconstruction results are shown in Fig. 4.

To obtain this reconstruction, 2160 sensors had to be placed along the boundary of the domain. The placement of such a number of sensors in the correct position may be impractical. This problem may be solved by using a third generation rotating scanner configuration, that is, for this particular example, 12 (that is, $6 \times 2$ ) sensors for the six $\rho$ values, rotated to 180 positions, could be used. For an alternative approach, based on interpolation, see also [34]. To assess the significance of uniform sampling in the $(\rho, \theta)$ space, we performed a second set of experiments, where the sensors were regularly placed along (a) Recovered field
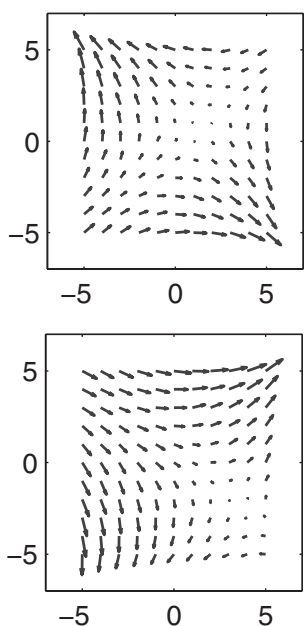

(b) Theoretical field
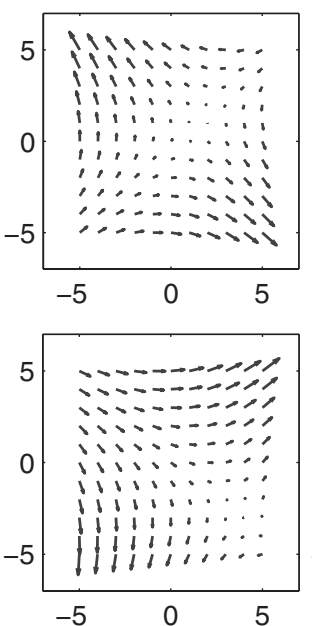

(c) Magnitude error (\%)

(d) Angular error $\left({ }^{\circ}\right)$
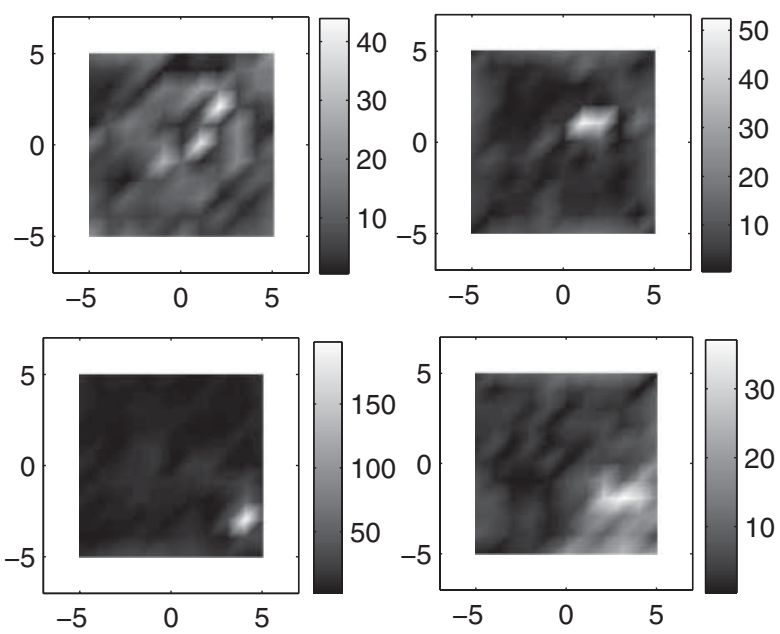

FIGURE 7. Simulation results for the reconstruction of some complicated fields. The reconstruction was based on integral data, the corresponding tracing lines which have uniform distribution in the Radon domain with $\Delta \rho=1$ and $\Delta \theta=2^{\circ}$. (a) The recovered vector field. (b) The electric field as computed from Coulomb's law. (c) The relative magnitude difference between the above two fields (\%). (d) The absolute angular difference (in degrees) between the directions of the recovered and the true field.

(a) Magn. err. (\%)

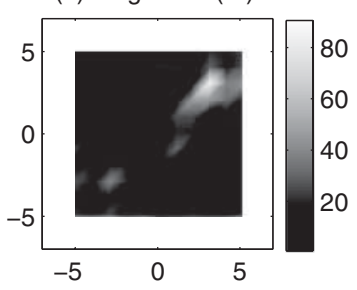

(b) Ang. err. ( ${ }^{\circ}$ )

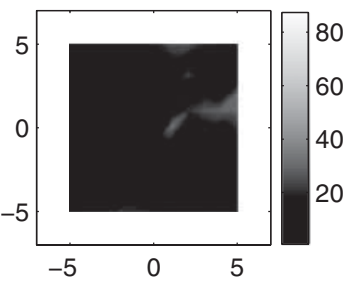

(c) Magn. err. (\%)

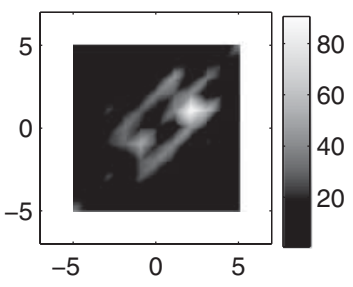

(d) Ang. err. $\left({ }^{\circ}\right)$

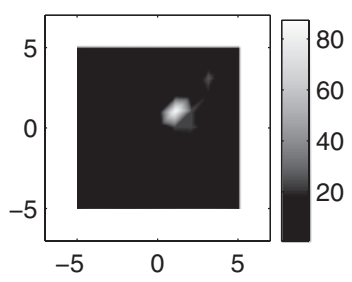

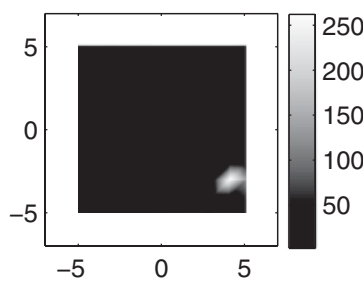
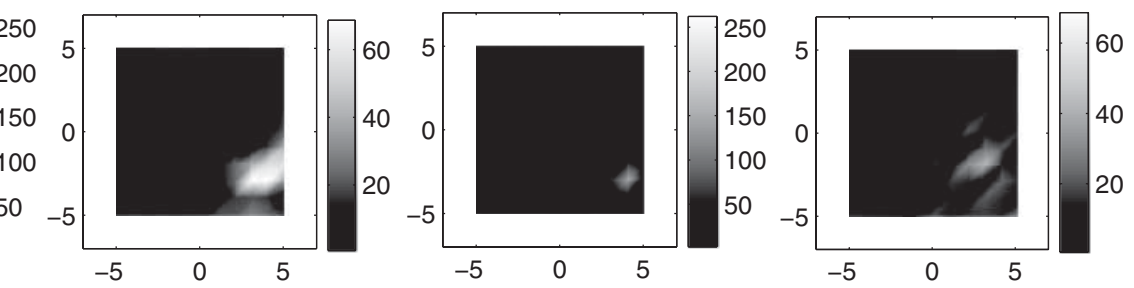

FIGURE 8. Errors in the reconstruction of some complicated fields. Left two columns: error in the reconstructions when the sensors are placed uniformly along the boundary. Right two columns: errors in the reconstruction when the sampling is done uniformly in the Radon domain with $\Delta \rho=1$ and $\Delta \theta=3^{\circ}$. 


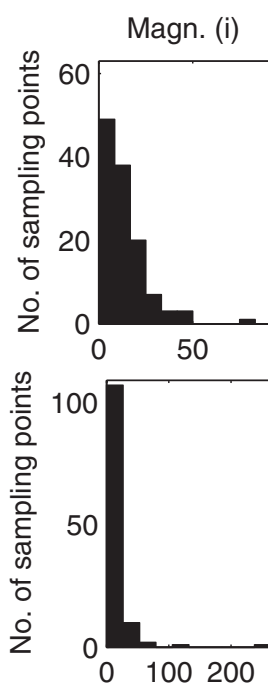

(\%)

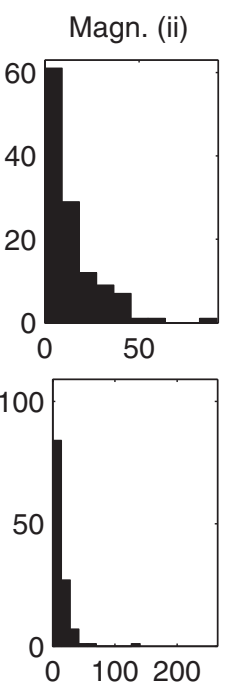

(\%)

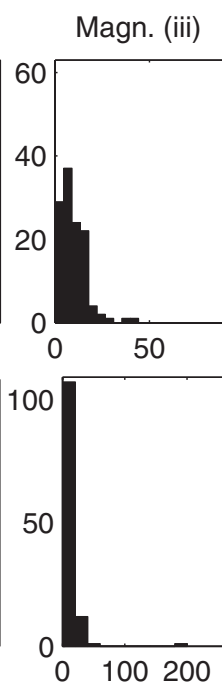

(\%)

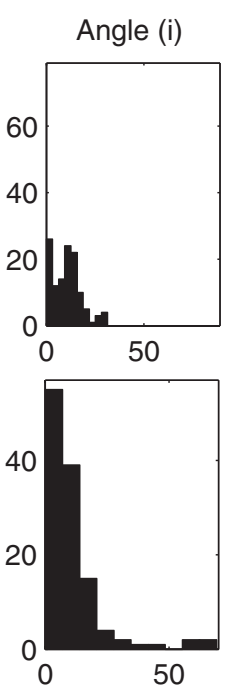

$\left({ }^{\circ}\right)$

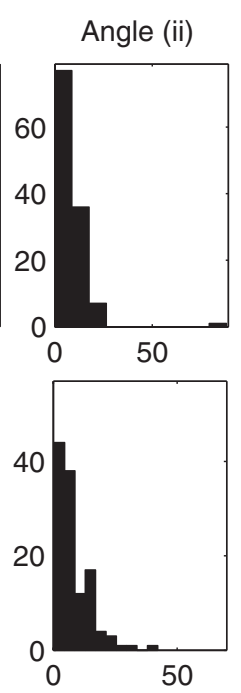

$\left({ }^{\circ}\right)$

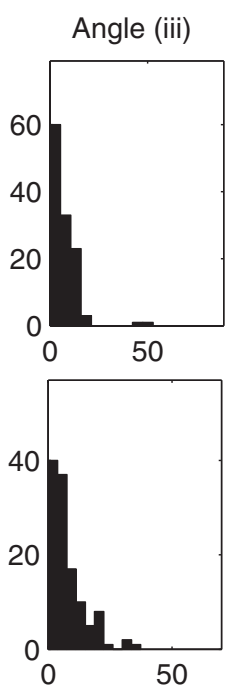

$\left({ }^{\circ}\right)$

FIGURE 9. Errors in the reconstruction of some complicated fields. Left three columns: the relative errors in magnitude for the cases (i) uniform sampling in $(x, y)$; (ii) uniform sampling in $(\rho, \theta)$ with $\Delta \rho=1$ and $\Delta \theta=3^{\circ}$; (iii) uniform sampling in $(\rho, \theta)$ with $\Delta \rho=1$ and $\Delta \theta=2^{\circ}$. Right three columns: the error in vector field orientation for the same cases.

the border of the domain, at the middle points of the boundary edges of all boundary tiles. Hence, we used 11 sensors on each side of the boundary of the square domain. We considered all possible voltage differences between pairs of these boundary points, apart from points lying on the same border line, and we formed the system of linear equations according to the description of Section 2. The number of available equations this time was 726, far fewer than for the previous case. In order to assess the effect of uniform sampling of the Radon space, we also run experiments with $\Delta \rho=1$ and $\Delta \theta=3^{\circ}$. This choice of sampling rates yields a system of $6 \times 120=720$ equations, comparable to that we obtain by placing the sensors uniformly along the domain border. The reconstructed fields for all positions of the static charge were visually indistinguishable from the reconstructions obtained by the first set of experiments. The error plots of both these sets of experiments are shown in Fig. 5. We note that the errors in the reconstruction are lower when the sampling is uniform in the $(\rho, \theta)$ space.

To appreciate better the improvement gained by using uniform sampling in the $(\rho, \theta)$ space, in Fig. 6 we present the histograms of the errors in each case. By close examination of Fig. 6, we may see that the use of uniform sampling in the $(\rho, \theta)$ space resulted in a much better vector field reconstruction. Note that in 14 out of the 16 panels referring to uniform sampling of the Radon space, the high-error bins are empty. In all cases, the average error is lower for uniform sampling of the Radon space. In particular, it was found that the average difference in vector field orientation measured in degrees was $27 \%$ lower when using uniform sampling in the Radon domain with $\Delta \rho=1$ and $\Delta \theta=2^{\circ}$, as opposed to uniform sampling in the $(x, y)$ domain, whereas the average error in magnitude was lower by $19 \%$.
The corresponding differences in angular and magnitude error for the case with $\Delta \rho=1$ and $\Delta \theta=3^{\circ}$, over the case of uniformity in the $(x, y)$ domain, were $20 \%$ and $13 \%$, respectively.

Figure 7 shows the results of the reconstruction of some much more complicated fields. These fields were produced by placing two point sources located at $(19,-19)$ and $(-16,21)$, and at $(-11,-24.5)$ and $(19,19)$. Figure 8 shows the errors of the reconstruction, and Fig. 9 the histograms of these errors. Note that the high reconstruction errors appear in areas where the field is almost 0 , i.e. in areas where in the calculation of the relative error we have a very small number in the denominator. Further, the effects of quantization noise become significant when the field is very weak and so its orientation is not calculated reliably. Note also that the error of the reconstruction using uniform sampling in the Radon space depends on the sampling rate used. For the derivation of the right sampling rates for a given problem, the reader is referred to [35].

\section{THE EFFECT OF NOISE ON THE RECONSTRUCTION}

In this section we investigate the effect of noise on the reconstruction of the vector field. In all experiments reported in the previous section, the sensors were placed exactly in the positions we had decided, and the measurement taken by each sensor was exactly the value predicted by Coulomb's law. In a practical system, however, some of the sensor measurements are expected to have inaccuracies and some of the sensor positions are also expected to be somehow inaccurate. To emulate these effects, we performed the following series of experiments. 
(i) We added a noise value to a measurement as a fraction of the true value, with random sign. For example, $2 \%$ noise means that the sensor measurement was changed by $2 \%$ of the value dictated by Coulomb's law. The change was either incremental or decremental, the choice made at random for each sensor.

(ii) We moved a sensor away from its true position by a fraction of the true position. For example, if according to the theory, a sensor should be placed at position $(x, y)$, and we consider a $2 \%$ error, then the coordinates of this sensor were shifted by $2 \%$ the corresponding correct values, with a positive or negative sign chosen at random.

(iii) We considered both the above errors simultaneously.

We performed four series of experiments: (a) we perturbed only $25 \%$ of the sensors; (b) we perturbed $50 \%$ of the sensors; (c) we perturbed $75 \%$ of the sensors; (d) all sensors were perturbed. In the cases where only some of the sensors were perturbed, the perturbed sensors were selected at random.

The source for all the simulations was located at $(19,-19)$. The sampling steps we used for the Radon domain were $\Delta \rho=1$ and $\Delta \theta=2^{\circ}$. (a)

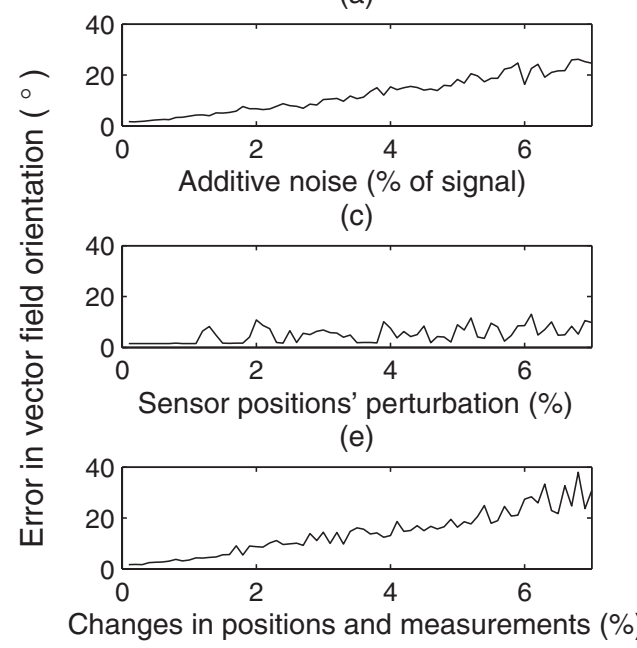

(b)

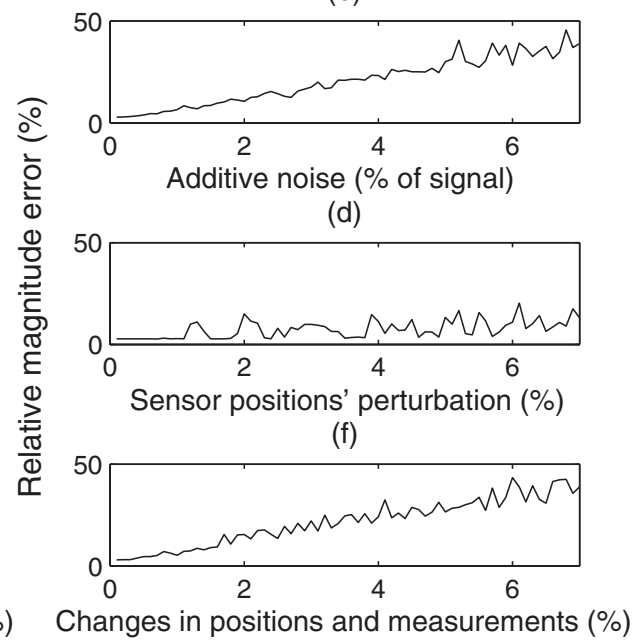

FIGURE 10. (a) and (b) Errors in vector field orientation and magnitude, when noise was added to the measurements of $25 \%$ of the sensors, as a percentage of the true value. (c) and (d) Errors in vector field orientation and magnitude, when small perturbations in the sensor positions were added. Position perturbations were a percentage of the true positions. (e) and (f) Errors in vector field orientation and magnitude, when both sensors' measurements and positions were changed by a percentage of their true values. In all cases, $25 \%$ of the sensors were perturbed.

(a)

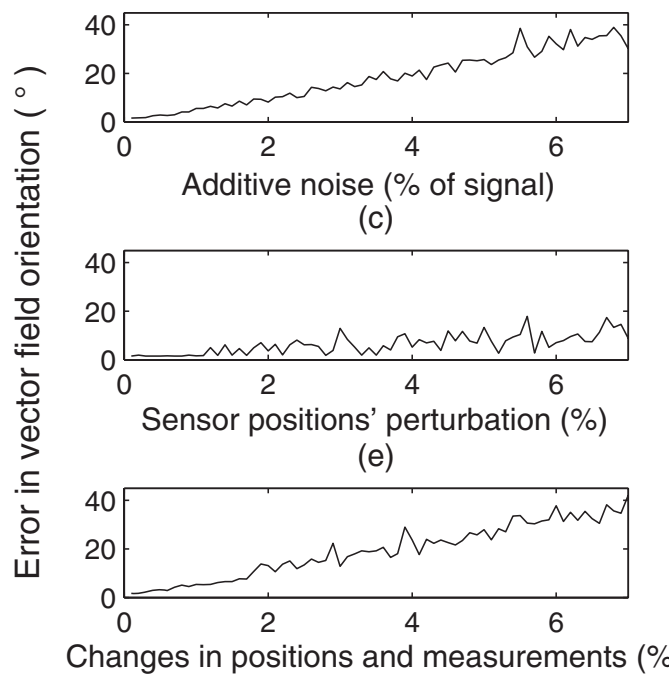

(b)

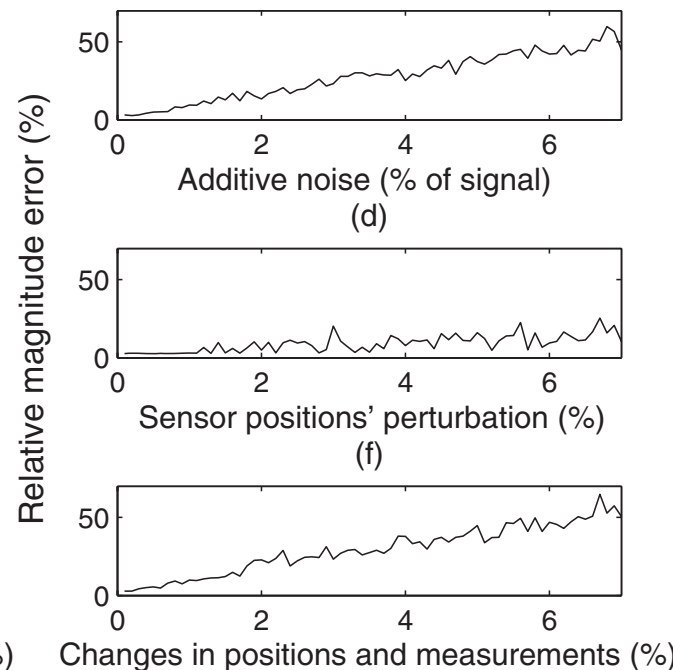

FIGURE 11. As in Fig. 10, but here 50\% of the sensors were perturbed. 
(a)

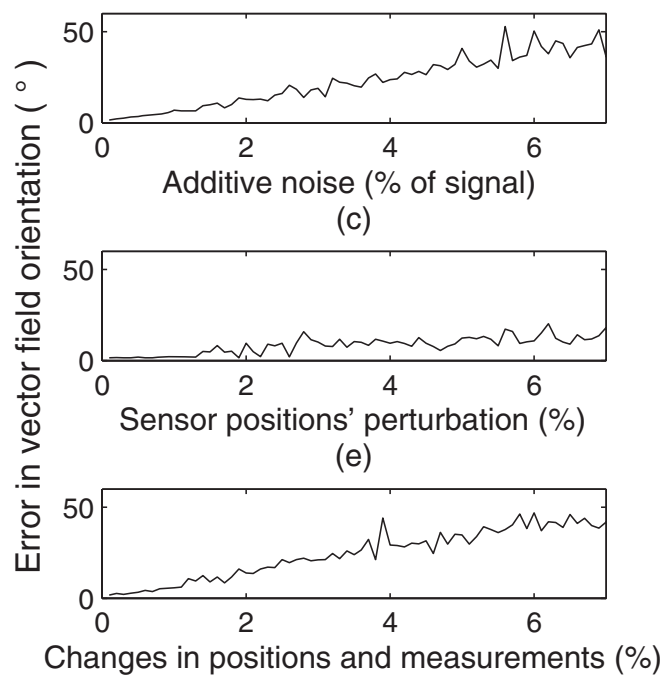

(b)

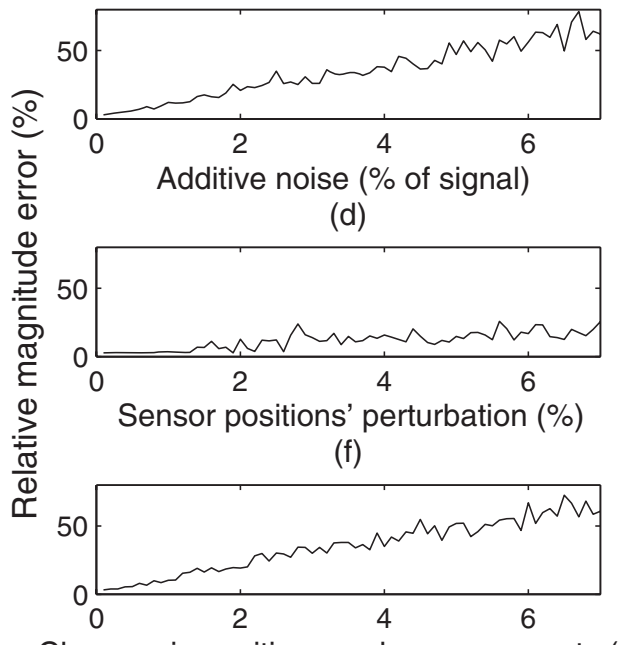

Changes in positions and measurements (\%)

FIGURE 12. As in Fig. 10, but here $75 \%$ of the sensors were perturbed.

(a)

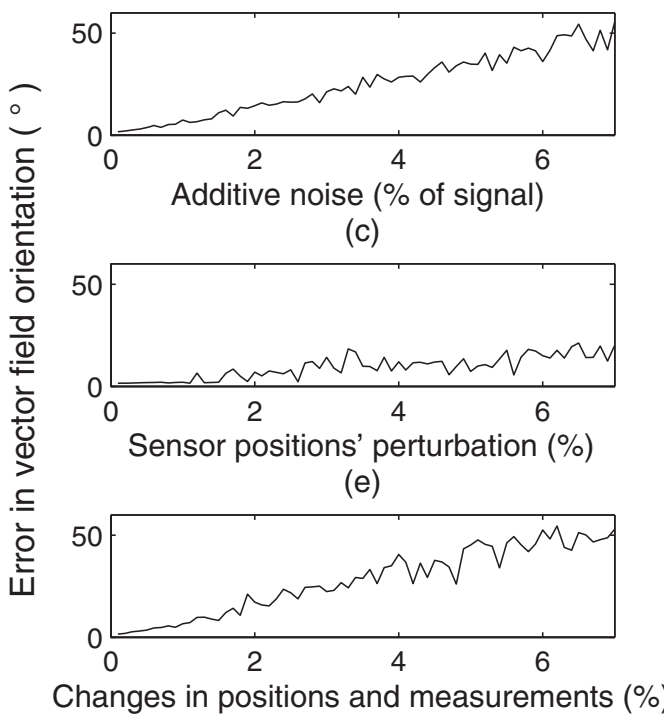

(b)

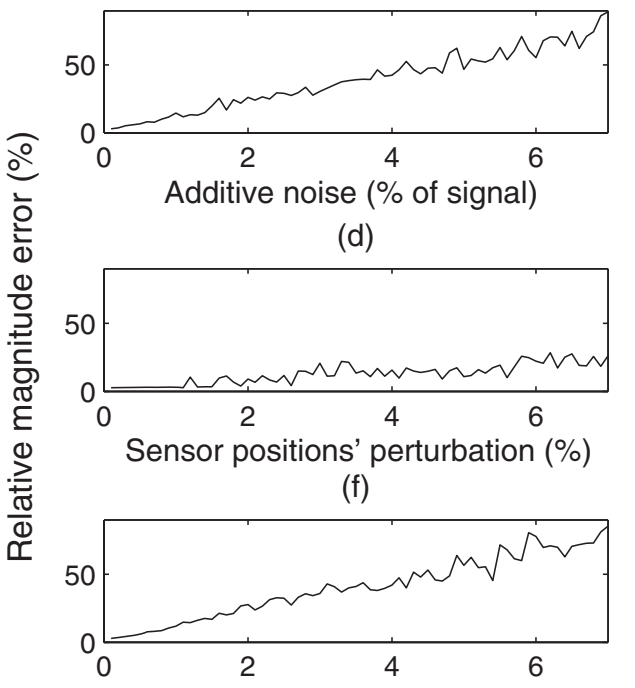

Changes in positions and measurements (\%)

FIGURE 13. As in Fig. 10, but here all sensors were perturbed.

The results of these experiments are shown in Figs 10-13. We observe that the results are relatively robust to perturbations in the position of the sensors, but much more sensitive to perturbations in the sensor measurements. This is not surprising as the difference in reconstruction accuracy was not very seriously affected either we were sampling uniformly in the Radon domain or we were placing the sensors uniformly along the border of the region.

The noise model assumed in these experiments is signal dependent. Noise processes of this type are inherent in many fields, such as optics [36], kinematics [37] and magnetic resonance imaging [38]. However, in many cases, for example in telecommunications, the noise that corrupts the data is signal independent. The implication of employing signal independent (additive or multiplicative) noise for the proposed algorithm is that the quality of reconstruction will be worse in places where the field is weak, and better in places where the field is strong.

\section{DISCUSSION AND CONCLUSIONS}

In this paper, the vector field tomography problem was discussed. In previous attempts to map integral measurements 
obtained along tracing lines onto a vector field, conventional (scalar) tomography theory had invariably been applied [7, 13, 14]: this had led to an under-determined problem. However, in this paper a new analysis was presented that aimed at the recovery of all components of a band-limited vector field at the sampling points of a 2D digitized bounded domain, by solving, this time, a system of linear equations. The reconstruction was based only on boundary integral measurements. We took advantage of the redundancy in boundary integral data with a view to recovering the vector field at discrete and finite in number sampling points, since these data may be seen as weighted sums of the local vector field's Cartesian components. One might think that the reconstruction errors may further be reduced if the contribution of each sampling point to the integral along a tracing line were taken into consideration in a more elaborate way, for example, by considering the length of each line segment inside the tile represented by the sampling point. This, however, would have made the solution of the problem much more complicated. The error introduced by the nearest neighbour interpolation we use is somehow dealt with by the redundancy in the linear system we solve, as the unknowns are generally much fewer than the equations. Indeed, one may consider replacing the nearest neighbour interpolation with bilinear or any other type of interpolation, where the distance of the line segment, along which we perform the integration, from the four nearest sampling points is taken into consideration. However, it was found that bilinear interpolation increased the angular error by $75 \%$ and the magnitude error by $70 \%$, compared with the nearest neighbour interpolation. We attribute this to the accumulation of projection errors, the fact that the system of linear equations becomes too complicated and the least-squares solution becomes more unpredictable.

An important issue when solving inverse problems is the sensitivity of the solution to noise. In the case of this problem, there are two possible sources of noise: inaccuracies in the sensor measurements and inaccuracies in the positions of the sensors. In a practical application, one may hope that one may use very accurate sensors and that even more accurate sensors may be developed in the future. The inaccuracies, however, in the sensor positions are rather intrinsic to the problem: the domain over which the vector field is to be reconstructed may not have a shape that helps the correct placement of the sensors. It is very encouraging, therefore, that the solution of the problem is relatively stable to perturbations in the sensor positions.

The solution is rather sensitive to the sensor measurements. For example, if only $25 \%$ of the sensors yield measurements that are only $4 \%$ wrong, the orientation angle of the reconstructed field is recovered with an average error of about $15^{\circ}$, while the magnitude of the reconstructed field is recovered with an average relative error of about $23 \%$. Such sensitivity to errors in the measurements may be overcome with the help of robust reconstruction methods. There are two ways to go about this. (i) One may solve the system of linear equations in a robust way. For example, instead of working out a solution that minimizes the sum of the squares of the errors with which individual equations are satisfied, one may use a robust redescending kernel [39] that will reduce the effect of outliers. The problem contains enough redundancy to permit such an approach.

(ii) The problem may be formulated as a Bayesian reconstruction problem [40], where a regularization term is added to a global cost function that expresses the adherence of the values of the reconstructed field to the obtained measurements. The regularization term may be such that it encourages the smooth variation of the field inside the domain. Expecting smooth field variation between neighbouring sampling positions is compatible with the assumption that there are no singularities inside the domain. Indeed, we consider this assumption pretty realistic as singularities usually arise due to poor mathematical modelling rather than being present in a physical system. Once a cost function of the solution has been formulated, it can be solved using Bayesian methods [41, 42] and a global optimization approach like, for example, simulated annealing [43].

The analysis performed here assumed that the sensors are placed in the desirable positions. However, in practice the user rarely has the option to place the sensors exactly at the desired positions. We have proposed elsewhere [34] the use of virtual sensors, placed at the optimal positions, where the 'measured' values are estimated from the true sensor values. Finally, we must emphasize that the reconstruction of the field in the digital domain is possible, only under the assumption that the field is band-limited. The presence of singularities in the domain of interest would result in a field with arbitrarily high frequencies, something that would have invalidated our method.

The proposed work may find application in CT angiography, i.e. in imaging blood vessel flow velocity. Further, it may be used to work out equivalent 'effective electrostatic fields' of complex systems, like the human brain, where the true electromagnetic field is very difficult to be reconstructed due, for example, to the different electromagnetic properties of the different types of tissue that are present in the anatomy of the brain. The idea is to consider the EEG measurements obtained by an EEG system with the maximum number of electrodes. Instead of trying to solve the inverse problem to identify the active regions of the brain, we may treat these measurements as the instantaneous voltages of an unknown electrostatic field, measured at the locations of the electrodes. We can then reconstruct this electrostatic field from these voltage values. This way we have a systematic way of mapping the true electromagnetic state of the brain to some space of electrostatic fields and follow its evolution in that space from the succession of samples collected. (For each combination of recorded values at fixed time, we shall have one electrostatic field.) These fields 
are not the true states of the human brain, but rather the states it would have had if it were made from tissue with uniform electromagnetic properties. That is why we call these states 'effective' and not true. We can then study the correlation between the sequences of the various effective states of the brain and the external stimuli or the actions the subject performs when the measurements are taken.

There are lots of practical issues that will have to be addressed in such applications. For example, in many practical situations, it is not possible to collect data over the complete angular range [3]. In the literature, this is referred to as the limited view problem. The reasons that cause this problem are varied. Limited data collection time, geometric constraints on the structure of the measurement apparatus, and the size and shape of the imaged object are some of the causes. Regarding the example we consider in this paper, it would not be possible in a practical situation to obtain measurements along lines that make an angle smaller than $20^{\circ}$ with each of the associated boundary edges, a situation that often arises in limited view tomographic reconstruction $[44,45]$. Then about $16 \%$ of the required line measurements would be missing. The limited angular coverage may cause several problems, the most serious of which is increased instability [46], which would increase sensitivity to noise. Another problem would be the possible non-uniqueness of the solution. To deal with such problems, many specialized algorithms have been introduced $[44,45]$. However, dealing with the solutions of these problems falls beyond the scope of this paper.

\section{FUNDING}

This work was supported by the 'Integrated Electronics' portfolio grant.

\section{REFERENCES}

[1] Hough, P.V.C. (1962) A Method and Means for Recognising Complex Patterns. US Patent, Vol. 3, 069, 654.

[2] Radon, J. (1917) Über die Bestimmung von Funkionen durch ihre Integralwerte Längs Gewisse Mannigfaltigkeiten. Berichte der Sächsischen Akademie der Wissenschaften (Leipzig). Math.Phys. Kl., 69, 262-277.

[3] Deans, S.R. (1993) The Radon Transform and Some of Its Applications (2nd edn). Krieger Publishing Company, Malabar, FL.

[4] Juhlin, S.P. (1993) Doppler Tomography. Proc. 15th Annual Int. Conf. IEEE Engineering in Medicine and Biology Society $(E M B C)$, San Diego, CA, USA, October 28-31, pp. 212-213.

[5] Tao, Y.K., Davis, A.M. and Izatt, J.A. (2008) Single-pass volumetric bidirectional blood flow imaging spectral domain optical coherence tomography using a modified Hilbert transform. Opt. Express, 16, 12350-12361.

[6] Howe, B.M., Worcester, P.F. and Spindel, R.C. (1987) Ocean acoustic tomography: mesoscale velocity, J. Geophys. Res., 92 , $3785-3806$.
[7] Munk, W. and Wunsch, C. (1982) Observing the ocean in the 1990s. Philos. Trans. R. Soc. Lond. A, Math. Phys. Sci., 307, 439-464.

[8] Rouseff, D., Winters, K.B. and Ewart, T.E. (1991) Reconstruction of oceanic microstructure by tomography: a numerical feasibility study. J. Geophys. Res., 96, 8823-8833.

[9] Johnson, S.A., Greenleaf, J.F., Tanaka, M. and Flandro, G. (1977) Reconstructing three-dimensional temperature and fluid velocity vector fields from acoustic transmission measurements. Instrum. Syst. Autom. Soc. Trans., 16, 3-15.

[10] Kramer, D.M. and Lauterbur, P.C. (1979) On the problem of reconstructing images of non-scalar parameters from projections. Applications to vector fields. IEEE Trans. Nucl. Sci., 26, 26742677.

[11] Norton, S.J. and Linzer, M. (1982) Correcting for ray refraction in velocity and attenuation tomography: a perturbation approach. Ultrason. Imaging, 4, 201-233.

[12] Norton, S.J. (1988) Tomographic reconstruction of 2D vector fields: application to flow imaging. Geophys. J. Int., 97, 161168.

[13] Norton, S.J. (1992) Unique tomographic reconstruction of vector fields using boundary data. IEEE Trans. Image Process., 1, 406412.

[14] Braun, H. and Hauck, A. (1991) Tomographic reconstruction of vector fields. IEEE Trans. Signal Process., 39, 464-471.

[15] Winters, K.B. and Rouseff, D. (1990) A filtered backprojection method for the tomographic reconstruction of fluid vorticity, Inverse Probl., 6, L33-L38.

[16] Winters, K.B. and Rouseff, D. (1993) Tomographic reconstruction of stratified fluid flow. IEEE Trans. Ultrason. Ferroelectr. Freq. Control, 40, 26-33.

[17] Zahn, M. (1994) Transform relationship between Kerr-effect optical phase shift and non-uniform electric field distributions. IEEE Trans. Dielectr. Electr. Insul., 1, 235-246.

[18] Hertz, H.M. (1986) Kerr effect tomography for nonintrusive spatially resolved measurements of asymmetric electric field distributions. Appl. Opt., 25, 914-921.

[19] Aben, H.K. (1987) Kerr effect tomography for general axisymmetric field. Appl. Opt., 26, 2921-2924.

[20] Efremov, N.P., Poluektov, N.P. and Kharchenko, V.N. (1995) Tomography of ion and atom velocities in plasmas. J. Quant. Spectrosc. Radiat. Transf., 53, 723-728.

[21] Faris G.W. and Byer, R.L. (1988) Three-dimensional beamdeflection optical tomography of a supersonic jet. Appl. Opt., 27, 5202-5212.

[22] Sharafutdinov, V.A. (1992) Tomographic problem of photoelasticity. Proc. Soc. Photo-Opt. Instrum. Eng., 1843, 234-243.

[23] Aben, H. and Puro, A. (1997) Photoelastic tomography for threedimensional flow birefringence studies. Inverse Probl., 13, 215221.

[24] Schwarz, A. (1994) Three-dimensional Reconstruction of Temperature and Velocity Fields in a Furnace. Proc. European Concerted Action on Process Tomography (ECAPT), Oporto, Portugal, March 24-26, 227-233.

[25] Segre, S.E. (1978) The measurement of poloidal magnetic field in a Tokamak by the change of polarization of an electromagnetic wave. Plasma Phys., 20, 295-307. 
[26] Juhlin, P. (1992) Principles of Doppler Tomography. LUTFD2/(TFMA-92)/7002+17P, Department of Mathematics, Lund Institute of Technology, Sweden, August.

[27] Rouseff, D. and Winters, K.B. (1994) Two-dimensional vector flow inversion by diffraction tomography. Inverse Probl., 10, 687-697.

[28] Petrou, M. and Wang, F. (2008) A Tutorial on the Practical Implementation of the Trace Transform, Chapter 11. In Mirmehdi, M. Xie, X. and Suri, J. (eds), Handbook of Texture Analysis. World Scientific, London.

[29] Petrou, M. and Kadyrov, A. (2004) Affine invariant features from the trace transform. IEEE Trans. Pattern Anal. Mach. Intell., 26, 30-44.

[30] Kadyrov, A. and Petrou, M. (2006) Affine parameter estimation from the trace transform. IEEE Trans. Pattern Anal. Mach. Intell., 28, 1631-1645.

[31] Schwarz, H.R. (1986) Numerische Mathematik. B. G. Teubner.

[32] Schwetlick, H. and Kretzschmar, H. (1991) Numerische Verfahren fur Naturwissenschaftler und Ingenieure. Fachbuchverlag.

[33] Bronshtein, I.N., Semendyayev, K.A., Musiol, G. and Muehlig, H. (2003) Handbook of Mathematics. Springer, New York.

[34] Giannakidis, A., Kotoulas, L. and Petrou, M. (2010) Virtual sensors for 2D vector field tomography. J. Opt. Soc. Am. A, 27, 1331-1341.

[35] Giannakidis, A. and Petrou, M. (2010) Sampling bounds for 2D vector field tomography. J. Math. Imaging Vis., 37, 151-165.

[36] Rangayyan, R.M., Ciuc, M. and Faghih, F. (1998) Adaptedneighborhood filtering of images corrupted by signal-dependent noise. Appl. Opt., 37, 4477-4487.
[37] Sanger, T.D., Kaiser, J. and Placek, B. (2005) Reaching movements in childhood dystonia contain signal-dependent noise. J. Child Neurology, 20, 489-496.

[38] Krüger, G. and Glover, G.H. (2001) Physiological noise in oxygenation-sensitive magnetic resonance imaging. Magn. Reson. Med., 46, 631-677.

[39] Hoaglin, D.C., Mosteller, F. and Tukey, J.W. (1983) Understanding Robust and Exploratory Data Analysis. Wiley.

[40] Mumcuoglu, E.U., Leahy, R., Cherry, S.R. and Zhenyu, Z. (1994) Fast gradient-based methods for Bayesian reconstruction of transmission and emission PET images. IEEE Trans. Med. Imaging, 13, 687-701.

[41] Arridge, S.R. and Simmons, A. (1997) Multi-spectral Probabilistic Diffusion using Bayesian Classification. Scale-Space Theory in Computer Vision. Lecture Notes in Computer Science 1252, Springer, pp. 224-235.

[42] Gindi, G., Lee, R. and Zubal, I.G. (1993) Bayesian reconstruction of functional images using anatomical information as priors. IEEE Trans. Med. Imaging, 12, 670-680.

[43] Kirkpatrick, S., Gelatt, C.D. and Vecchi, M.P. (1983) Optimization by simulated annealing. Science, 220, 671-680.

[44] Delaney, A.H. and Bresler, Y. (1998) Globally convergent edgepreserving regularised reconstruction: an application to limited angle tomography. IEEE Trans. Image Process., 7, 204-221.

[45] Reeds, J.A. and Shepp, L.A. (1987) Limited angle reconstruction in tomography via squashing. IEEE Trans. Med. Imaging, 6 , 89-97.

[46] Natterer, F. and Wubbeling, F. (2001) Mathematical Methods in Image Reconstruction. SIAM, Philadelphia, PA. 